\title{
Triploid Wild Rice (BKK) Strain Found in Bangkok Originated from Hybridizations among Three Parental Oryza Species
}

\author{
So Makabe', Htet Aung Htut ${ }^{2}$, Hiroko Takahashi' ${ }^{1}$, Sayaka Shida², Masahiro Akimoto ${ }^{3}$, \\ Hathairat Urairong ${ }^{4}$, Ryuji Ishikawa ${ }^{5}$, Tadashi Sato6, Yo-Ichiro Sato ${ }^{7}$, Ikuo Nakamura2*
}

\author{
${ }^{1}$ BEX Co., Ltd., Tokyo, Japan \\ ${ }^{2}$ Graduate School of Horticulture, Chiba University, Matsudo, Japan \\ ${ }^{3}$ Obihiro University of Agriculture and Veterinary Medicine, Obihiro, Japan \\ ${ }^{4}$ Biotechnology Research and Development Office, DOA, Thanyaburi, Thailand \\ ${ }^{5}$ Faculty of Agriculture and Life Science, Hirosaki University, Hirosaki, Japan \\ ${ }^{6}$ Graduate School of Life Sciences, Tohoku University, Sendai, Japan \\ ${ }^{7}$ Kyoto Prefectural University, Kyoto, Japan \\ Email: ^inakamur@faculty.chiba-u.jp
}

How to cite this paper: Makabe, S., Htut, H.A., Takahashi, H., Shida, S., Akimoto, M., Urairong, H., Ishikawa, R., Sato, T., Sato, Y.-I. and Nakamura, I. (2022) Triploid Wild Rice (BKK) Strain Found in Bangkok Originated from Hybridizations among Three Parental Oryza Species. American Journal of Plant Sciences, 13, 36-49. https://doi.org/10.4236/ajps.2022.131003

Received: November 29, 2021

Accepted: January 11, 2022

Published: January 14, 2022

Copyright $\odot 2022$ by author(s) and Scientific Research Publishing Inc. This work is licensed under the Creative Commons Attribution International License (CC BY 4.0).

http://creativecommons.org/licenses/by/4.0/

\section{(c) (i) Open Access}

\begin{abstract}
A wild rice (BKK) strain showing sterile spikelet and big leaves inhabited at the basin of the Chao Phraya river of Bangkok city, Thailand. The BKK strain was found as a natural triploid and thus its origin has been interested long time. Three different-sized fragments were amplified in RNA polymerase I largest subunit (PolA1) gene, which is a single-copy nuclear gene per haploid genome. Short type $(0.14 \mathrm{~kb})$ intron 20 sequence of BKK strain was identical to that of $O$. rufipogon and $O$. sativa. Phylogenetic analysis showed that long type $(1.5 \mathrm{~kb}$ and $1.8 \mathrm{~kb})$ intron 20 sequences of BKK strain were closely related to that of $O$. longistaminata and $O$. officinalis, respectively. We analyzed protein tag (Ptag) sequence encoded by exons 19 to 21 of PolA1 gene. Determined three Ptag sequences of BKK strain were identical to that of $O$. rufipogon, $O$. longistaminata, and $O$. officinalis, respectively. Relative DNA content of nuclei in $O$. officinalis and BKK strain was 1.5 and 1.75 times than that in O. sativa, respectively. And BKK strain contained CentO-C1 repeats, which were unique to $O$. officinalis. These results indicated that BKK strain comprised three genomes of $O$. rufipogon, $O$. longistaminata, and $O$. officinalis.
\end{abstract}

\section{Keywords}

Oryza rufipogon, Triploid, Flow Cytometry, PolA1, CentO-C1 Repeat Sequence 


\section{Introduction}

During several rounds of wild rice surveys, initiated by Drs. Morishima and Oka in 1958, a wild rice (BKK) strain had been found in canals, near the Wat Chalo temple, from the Chao Phraya river in the Bang Kruai district (described as Bangkok Noi), Bangkok, Thailand (Figure 1). The clonally propagated BKK strain with sterile spikelet had unusually big leaves, leaf width: more than $3.5 \mathrm{~cm}$, leaf length: more than $1 \mathrm{~m}$, which are several times bigger than those of $O$. rufipogon (Figure 1). Because W0001 (O. ridleyi) and W0002 (O. officinalis) strains of wild rice germplasm in the National Institute of Genetics, Japan was obtained in the same place, we are being interested in the origin of BKK strain.

Chu and Oka (1970) reported that hybrids between O. longistaminata (formally described as $O$. barthii) and other species showed off-types with big leaves, which they called "Obake" (a monster in Japanese) [1]. In the Mekong Delta of Viet Nam, we found another wild rice strain with big leaves, which was probably a hybrid between $O$. rufipogon and cryptic $O$. longistaminata (Htut et al. submitted). The BKK strain, however, had bigger leaves than these wild rice strains mentioned above.

The BKK strain was consistent with O. rufipogon and O. officinalis in Bangkok in habitat (Figure 1). Although O. longistaminata and O. barthii are distributed in West Africa, we recently found O. longistaminata-like cryptic (MD) strain in the Mekong Delta (Htut et al. submitted). Other AA genome wild species, O. meridionalis and O. glumaepatula are endemic in Australia and Latin America, respectively. Takahashi et al. (2009) reported based on sequence analysis

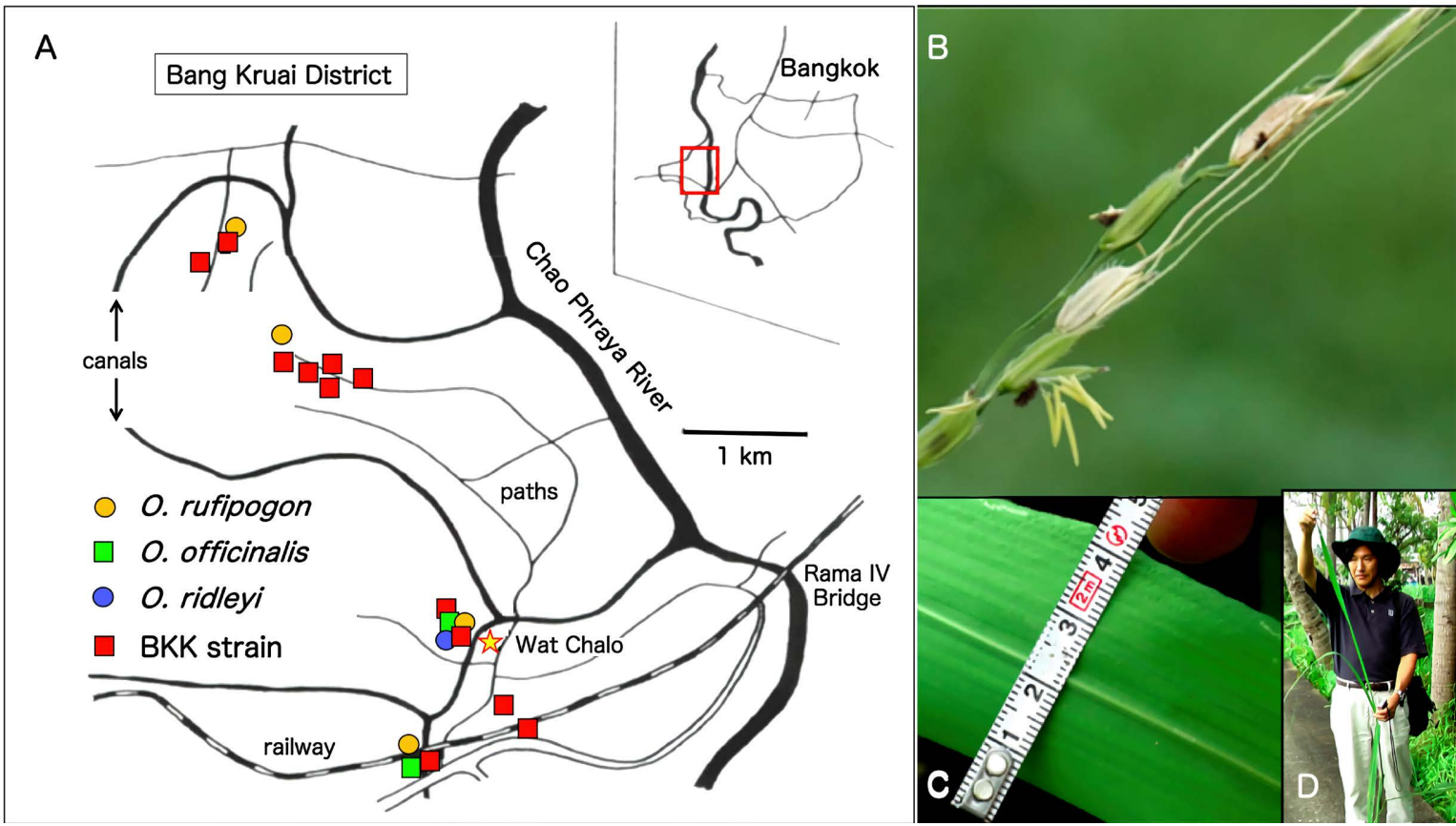

Figure 1. Habitat of BKK strain. (A) localization of three wild rice species (O. rufipogon, O. officinalis and O. ridleyi) and BKK strain near Wat Chalo in Bang Kruai district of Bangkok city in Thailand. Sterile spikelet (B), Leaf width (C), and Leaf length (D) of BKK strain. 
of nuclear gene that AA genome species was closely related to $\mathrm{BB}$ and $\mathrm{CC}$ genome species and distantly related to EE, FF and GG genome species [2]. The same results were obtained by the analysis of retrotransposons [3].

Genome sizes of 14 Oryza diploid species containing 6 different genome-types (AA, BB, CC, EE, FF, and GG) were measured using flow cytometry [4]. Except for $\mathrm{CC}$ genome, relative DNA content of nuclei corresponding to genome size was conservative characteristics to each genome-type. Thus, the DNA content is helpful to infer genome composition of the BKK strain.

Single-copy nuclear gene, PolAl, encodes the largest subunit (194 kDa) of RNA polymerase I which plays an essential role in the synthesis of 45S rRNA precursors [5]. The PolA1 gene (ca. $15 \mathrm{~kb}$ ) contains 21 exons on chromosome 6 of rice genome [6]. Phylogenetic studies for the intron 19 and exon 20 sequences of PolA1 gene were useful to analyze relationships in Petunia [7], Aegilops [8], Oryza [2], Triticum-Aegilops [9], Triticum-Hordeum [10], and Brassica [11] [12].

Recently, we found that intron 20 sequence of PolA1 gene were differentiated into short (S)-type $(0.14 \mathrm{~kb})$ and long $(\mathrm{L})$-type $(1.5 \mathrm{~kb})$ in Oryza AA-genome species. $O$. longistaminata and $O$. meridionalis had L-type intron 20 while $O$. rufipogon, $O$. barthii, $O$. glumaepatule contained S-type intron 20. Because $O$. officinalis and other Oryza species had the L-type, this result provided evidence for the speciation from L-type species to S-type species within AA genome species (Htut et al. submitted).

Nakamura (2016) found that a particular protein tag (Ptag) sequence (ca. 400 aa) showed a species-specific variation, encoded by exons 19 - 21 of PolA1 gene in land plants [13]. The Ptag sequence is useful to classify species in Triticum-Hordeum [10], Brassica [12], and Trichophyton fungi [14]. Therefore, Ptag sequences of BKK strain were determined to reveal species, which were involved in the origin of BKK strain.

In this paper, we are interested in the origin of natural triploid BKK strain found in Bangkok city. We found that BKK strain contained three different introns 20 sequences of PolA1 gene and Ptag-coding sequences, corresponding to those of $O$. rufipogon, $O$. longistaminata and $O$. officinalis, respectively. The results of relative DNA content of leaf cells and Southern blot analyses of CentO-C1 repeats also suggested that $O$. officinalis probably provided its genetic materials to BKK strain.

\section{Materials and Methods}

\subsection{Plant Materials}

BKK strain was collected in Bangkok city at 1985 and maintained in Japan (Figure 1). Other plant materials were obtained from National Institute of Genetics, Japan (Table 1).

\subsection{Chromosome Counting}

Chromosome number of root-tip cells of BKK strain was counted by enzyme maceration/air drying and Giemsa staining according to Fukui (1996) [15]. 
Table 1. Materials to analyze the intron 20 sequence of PolA1 gene in Oryza species.

\begin{tabular}{|c|c|c|c|c|c|}
\hline Species & Accession & Description & Intron 20 & Genome & $\mathrm{A} / \mathrm{P}$ \\
\hline \multirow{3}{*}{ O. sativa } & \multicolumn{2}{|c|}{ 'Nipponbare' Temperate Japonica } & $141 \mathrm{bp}$ & $\mathrm{AA}$ & \\
\hline & Ac221 & Tropical Japonica & $141 \mathrm{bp}$ & AA & \\
\hline & Ac130 & Indica & $141 \mathrm{bp}$ & AA & \\
\hline \multirow[t]{4}{*}{ O. rufipogon } & W0106 & India & $141 \mathrm{bp}$ & $\mathrm{AA}$ & A \\
\hline & W0107 & India & $141 \mathrm{bp}$ & $\mathrm{AA}$ & A \\
\hline & W1724 & India & $141 \mathrm{bp}$ & AA & $\mathrm{P}$ \\
\hline & W1956 & China & $141 \mathrm{bp}$ & AA & $\mathrm{P}$ \\
\hline \multirow[t]{2}{*}{ O. barthii } & W0652 & Sierra Leone & $142 \mathrm{bp}$ & AA & A \\
\hline & W1416 & Sierra Leone & $142 \mathrm{bp}$ & AA & A \\
\hline \multirow[t]{2}{*}{ O. longistaminata } & W0643 & Gambia & 1499 bp & AA & $\mathrm{P}$ \\
\hline & W1232 & Tanganyika & 1523 bp & AA & $\mathrm{P}$ \\
\hline \multirow[t]{2}{*}{ O. meridionalis } & W1297 & Australia & 1519 bp & AA & A \\
\hline & W1631 & Australia & $1523 \mathrm{bp}$ & AA & A \\
\hline \multirow{2}{*}{ O. glumaepatula } & W1169 & Cuba & $141 \mathrm{bp}$ & AA & $\mathrm{P}$ \\
\hline & W1185 & Suriname & $141 \mathrm{bp}$ & AA & $\mathrm{P}$ \\
\hline O. punctata & W1514 & Kenya & 1485 bp & $\mathrm{BB}$ & $\mathrm{P}$ \\
\hline O. officinalis & W0002 & Thailand & 1764 bp & $\mathrm{CC}$ & $\mathrm{P}$ \\
\hline O. eichingeri & W1521 & Uganda & 1767 bp & $\mathrm{CC}$ & $\mathrm{P}$ \\
\hline O. rhizomatis & W1808 & Sri Lanka & 1766 bp & $\mathrm{CC}$ & $\mathrm{P}$ \\
\hline \multirow[t]{2}{*}{ O. australiensis } & W0008 & Australia & $1103 \mathrm{bp}$ & $\mathrm{EE}$ & $\mathrm{P}$ \\
\hline & W1296 & Australia & $1100 \mathrm{bp}$ & $\mathrm{EE}$ & $\mathrm{P}$ \\
\hline O. brachyantha & W0656 & Guinea & $2643 \mathrm{bp}$ & $\mathrm{FF}$ & $\mathrm{P}$ \\
\hline \multirow[t]{2}{*}{ O. granulata } & W0003 & India & $1634 \mathrm{bp}$ & GG & $\mathrm{P}$ \\
\hline & W0004 & India & 1634 bp & GG & $\mathrm{P}$ \\
\hline \multirow[t]{3}{*}{ BKK strain } & & Thailand & $141 \mathrm{bp}$ & & $\mathrm{P}$ \\
\hline & & & $1519 \mathrm{bp}$ & & \\
\hline & & & $1765 \mathrm{bp}$ & & \\
\hline
\end{tabular}

A/P: annual/perennial.

For example, the head margin in this template measures proportionately more than is customary. This measurement and others are deliberate, using specifications that anticipate your paper as one part of the entire journals, and not as an independent document. Please do not revise any of the current designations.

\subsection{Measurement of Relative DNA Content}

Relative nuclear DNA contents of nuclei of leaf cells were determined by flow cytometry using a PA flow cytometer (Partec $\mathrm{GmbH}$, Münster, Germany) on isolated nuclei stained with 4',6-diamidino-2-phenylindole dihydrochloride 
(DAPI), according to the method of Mishiba et al. (2000) [16].

\subsection{DNA Extraction}

Total genomic DNA was extracted from $100 \mathrm{mg}$ leaf materials in 2-ml plastic tubes that were frozen with liquid nitrogen and crushed into fine powder using Multi-Bead Shocker (Yasui Kikai Co., Japan). The CTAB method was used for DNA extraction [17].

\subsection{PCR Amplification and Direct Sequencing}

As shown in Figure 3(A), DNA fragments containing Ptag-coding sequence and intron 20 (S-type and L-type) were amplified by PCR using various pairs of eleven primers (Table 2). The primers were designed based on the sequence of rice PolA1 mRNA (LOC9270399) on japonica rice "Nipponbare". Sequencing primers (5 - 8) was designed based on sequence result of the intron 20 in this study. Two plastid regional sequences, ORF100 (676 bp) and psbZ (792 bp), of BKK strain were determined using primer $11-14$ shown in Figure 4(B) according to Takahashi et al. (2009) [18].

The PCR amplification was carried out ExTaq DNA polymerase (TaKaRa, Shiga, Japan) according to manufacturer's instruction. The PCR condition were

Table 2. Primers used in this study.

\begin{tabular}{|c|c|c|}
\hline Name & Sequence & Description \\
\hline 1 & 5’-CTCGCTGGACGGGGTGAGATGAATG-3’ & PolA1, exon $195 \mathrm{P}$ \\
\hline 2 & 5'-CCTTGAGAACTGTTTTTATTGATG-3' & PolA1, exon 20 5P1 \\
\hline 3 & 5'-GAGCAACCTCATATTCTGTTAGCC-3' & PolA1, exon 20 5P2 \\
\hline 4 & 5'-CTGTGCATACTTCAATTCTCTC-3' & PolA1, exon $203 \mathrm{P} 1$ \\
\hline 5 & 5'-ТСТАAАСАТАТАСТСССТССАТСС-3' & $\begin{array}{l}\text { PolA1, intron } 205 \mathrm{P} \text { for } \\
\text { AA genome species }\end{array}$ \\
\hline 6 & 5'-AACATATACTACACTTATCTTACC-3' & $\begin{array}{l}\text { PolA1, intron } 205 \mathrm{P} \text { for } \mathrm{CC} \\
\text { genome species }\end{array}$ \\
\hline 7 & 5'-АСААСТТСТССАССААСАТТСТСТ-3' & $\begin{array}{l}\text { PolA1, intron } 203 \mathrm{P} \text { for } \mathrm{AA} \\
\text { genome species }\end{array}$ \\
\hline 8 & 5'-TAAAGGAATATCATATCAAAACAG-3' & $\begin{array}{l}\text { PolA1, intron } 203 \mathrm{P} \text { for } \mathrm{CC} \\
\text { genome species }\end{array}$ \\
\hline 9 & 5'-CTTACAGGCCTTGACAAAAACAGA-3' & PolA1, exon $213 \mathrm{P} 2$ \\
\hline 10 & 5’-TGAAATCCGCAATCAAGTTCAGATG-3' & PolA1, exon $213 \mathrm{P} 1$ \\
\hline 11 & 5'-GCCGCTTTAGTCCACTCAGCCATC-3' & ORF100 5P \\
\hline 12 & 5'TCAATGCCTTTTTTCAATGGTCTC-3' & ORF100 3P \\
\hline 13 & 5'-TATTTGCTTCTCCTGATGGTTGTT-3' & $p s b Z 5 \mathrm{P}$ \\
\hline 14 & 5'-GAGCGGAGTAGAGCAGTTTGGTAG-3’ & $\operatorname{trn} M 3 \mathrm{P}$ \\
\hline 15 & 5'-GTGTAAAAGTTATGTTTCACAAAT-3' & CentO-C1 5P \\
\hline 16 & 5'-CGGTGTGCCCGCTGGAAAGTTTGT-3' & CentO-C1 3P \\
\hline
\end{tabular}


40 cycles of $94^{\circ} \mathrm{C}$ for $1 \mathrm{~min}, 58^{\circ} \mathrm{C}$ for $1 \mathrm{~min}$ for annealing, and $72^{\circ} \mathrm{C}$ for $2 \mathrm{~min}$ for elongation in a PTC200 thermocycler (MJ Research, Waltham, MA, USA). The amplified PCR products were subjected to $1.5 \%$ agarose gel electrophoresis and purified using a PCR purification kit (QIAquick; Qiagen, CA, USA). The purified PCR products of Ptag-coding sequence (1230 bp) were determined by direct sequencing with the same primer as used for PCR amplification in an automated DNA sequencer ABI3100 (Applied Biosystems, CA, USA) with a Big Dye Terminator Cycle Sequencing kit (Applied Biosystems, USA).

\subsection{Phylogenetic Analysis}

The intron 20 sequences of PolA1 gene were aligned by using CLUSTAW [19]. The alignment was then manually adjusted using Genetyx Software ver. 6.0, Software Development Co., Japan. The phylogenetic tree of the intron 20 sequences were constructed using Neighbor-Joining method with bootstrap estimate from 1000 replicates in the MEGA7 software [20]. The determined intron 20 and Ptag-coding sequences of PolA1 gene were deposited in DDBJ as accession nos. (LC638415-LC638446).

\subsection{Southern Blot Analysis of Cent0-C1 Repeats}

CentO-C1 repeat sequence $(1.2 \mathrm{~kb})$ was amplified by PCR with DNA extracted from $O$. officinalis as a template using primer 15 and 16 (Table 2). Primers were designed based on sequence of CentO-C1_11 (Genbank accession DQ058478) [21]. Genomic DNA was extracted from leaves using the cetyl trimethyl ammonium bromide (CTAB) method according to Rogers and Bendich (1985) [22]. Genomic DNAs $(15 \mu \mathrm{g})$ extracted from leaves of $O$. sativa, $O$. officinalis and BKK strain were digested overnight with HindIII, EcoRI and $K p n I$ at $37^{\circ} \mathrm{C}$.

Restricted DNA fragments were separated on $0.7 \%$ agarose gel at $50 \mathrm{~V}$ for 4 hours and transferred to a nylon membrane overnight by capillary method. Fixing of DNA to membrane was done by exposure under a UV transilluminator for $3 \mathrm{~min}$. CentO-C1 fragment was labelled by PCR reaction incorporating a digoxigenin-labelled nucleotide as in PCR DIG Probe Synthesis Kit (Roche) protocol. Hybridization and stringency washes were carried out following the DIG manual while detection was done by chemiluminescence using CDP-Star, according to manufacturer's instructions.

\section{Results}

\subsection{Habitat of BKK Strain}

BKK strain was found in canals near the Wat Chalo temple together with three perennial Oryza species, O. rufipogon, O. officinalis and O. ridleyi (Figure 1(A)). The BKK strain was clonally propagated by shooting from each node of its column. Because the BKK strain had big stature with completely sterile spikelet (Figure 1(B)) and long-wide leaf blades (Figure 1(C), Figure 1(D)), it was considered as an interspecific hybrid between Oryza species. 


\subsection{Chromosome Number and Relative DNA Content}

Chromosome number of BKK strain was confirmed as 36 using root-tip cell (Figure 2(A)). This result clearly indicated that BKK strain was triploid. Relative nuclear DNA content of leaf cells was determined by flow cytometer analysis (Figure 2(B), Figure 2(C)). In case of relative DNA content of $O$. sativa was adjusted to $100, O$. officinalis showed a value of 150 (Figure $2(\mathrm{~B})$ ). In the same condition, BKK strain showed a value of 175 , that was probably corresponded to two haploid A genome $(50+50)$ and one haploid C genome (75) (Figure 2(C)).

\subsection{Analysis of PolA1 Intron 20 Sequence in BKK Strain}

PCR product containing intron 20 of PolA1 gene was amplified using primer 3 and 10 (Figure 3(A)). Short (S)-type intron $20(0.14 \mathrm{~kb})$ was shared with japonica and indica strains of $O$. sativa and two strains of $O$. rufipogon whereas $O$. longistaminata and $O$. officinalis showed long (L)-type intron $20(1.5 \mathrm{~kb}, 1.8 \mathrm{~kb})$ (Figure 3(B)). BKK strain contained one S-type intron 20 (rufA) and two L-type intron 20 (lonA, offC) (Figure 3(B)). Sequence of the S-type intron 20 was identical to that of $O$. rufipogon and $O$. sativa (Htut et al. submitted).

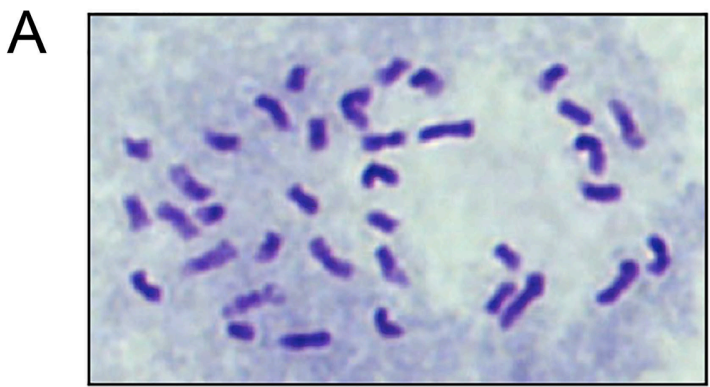

Chromosome of BKK strain $(n=36)$
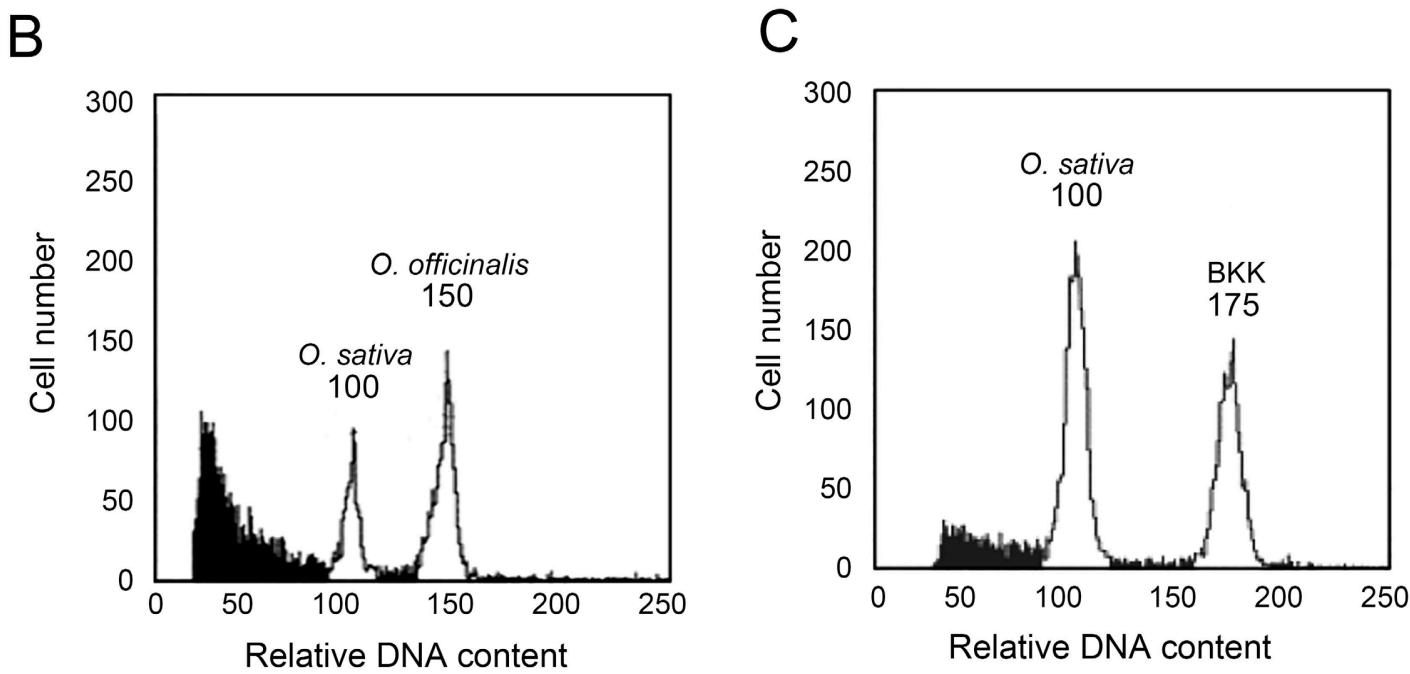

Figure 2. Chromosome number and relative DNA content of BKK strain. (A) Chromosomes $(n=36)$ of root-tip cell in BKK strain, (B) Relative DNA content of leaf cells in $O$. officinalis was measured using flow cytometry. As internal control, relative DNA content of $O$. sativa was adjusted to 100 . (C) Relative DNA content of leaf cells in BKK strain. 


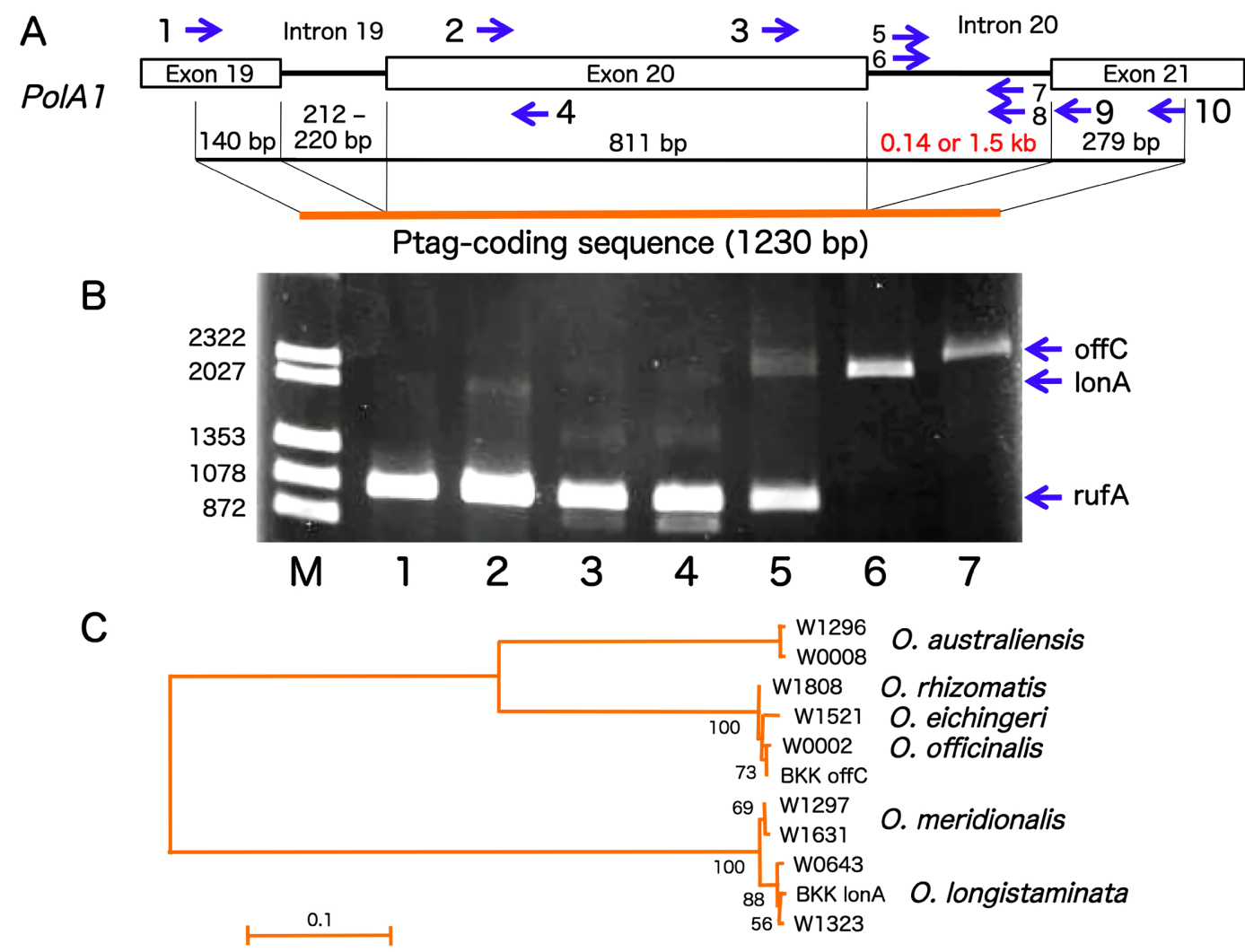

Figure 3. Analysis of intron 20 sequences of PolA1 gene in BKK strain. (A) Schematic diagram showing the position of primers $(1-10)$ used for PCR amplification and sequencing of intron 20 sequence of PolA1 gene (Table 2). Long (L)-type intron 20 sequences $(1.5 \mathrm{~kb}$ ) of AA genome species were determined using a pair of primer 5 and 7, whereas L-type intron 20 sequence $(1.8 \mathrm{~kb})$ of CC genome species were determined using a pair of primer 6 and 8. Three Ptag-coding sequences of BKK strain were also analyzed using primers shown in Figure 3(A). (B) PCR amplicons containing the intron 20 sequences in Oryza diploid species. M: Size marker $\lambda /$ HindIII and $\varphi$ x174/HaeIII, 1: O. sativa subsp. japonica, 2: N16 O. sativa subsp. indica, 3: O. rufipogon W0593, 4: O. rufipogon W1236, 5: BKK strain showed three bands (offC, lonA, and rufA), 6: O. longistaminata IRGC101205, 7: O. officinalis. (C) Neighbor-joining phylogenetic tree was constructed by using MEGA7 software based on the L-type intron 20 sequences among two AA genome species, three CC genome species, and BKK strain.

Sequences of two L-type intron 20 of BKK strain were analyzed using sequencing primer 5 and 7 specific to $O$. longistaminata and primer 6 and 8 specific to $O$. officinalis (Figure 3(A)). Neighbor-Joining phylogenetic tree of L-type intron 20 sequences was constructed (Figure 3(C)). One L-type tntron 20 sequence (lonA) of BKK strain was clustered with those of O. longistaminata and $O$. meridionalis (AA genome species) while another L-type intron 20 sequence (offC) was grouped with those of $O$. officinalis, $O$. eichingeri and $O$. rhizomatis (CC genome species).

\subsection{Comparison of Ptag-Coding Sequences in BKK Strain}

Three Ptag-coding sequences (1230 bp) of BKK strain were determined using primers shown in Figure 3(A). Alignment of three deduced Ptag sequences showed that two amino acid substitutions were found between BKK1 and BKK2 
but BKK1 and BKK3 were differed in 16 substitutions (Figure 4(A)). Interestingly, BKK1, BKK2 and BKK3 of BKK strain were identical to that of O. rufipogon, $O$. longistaminata and $O$. officinalis, respectively.

\subsection{Analysis of Two Plastid Sequences in BKK Strain}

Two regional plastid sequences of ORF100 (676 bp) and psbZ (792 bp) were compared among BKK strain, O. longistaminata, O. rufipogon and $O$. officinalis (Figure 4(B)). Sequences of BKK strain were different from those of $O$. officinalis and similar to those of $O$. rufipogon and $O$. longistaminata. Perennial strain of $O$. rufipogon and japonica strain of $O$. sativa contained an unique (GGGA) insertion in $p s b Z$ sequence.

\subsection{Southern Blot Analysis of Cent0-C1}

CentO-C1 repeat sequences specific to $O$. officinalis were amplified by PCR according to Lee et al. (2005) [21]. Genomic DNA isolated from O. sativa, $O$.

A BKK1 LKELLMTASAKISTPFMKCPLLEDKTWDDDEEEMDDKLKKARDAERLAAKLRTIDDAERIAAKLRRVRVADIVERIEVCTVPFHNNNGCV BKK2 LKELLMTASAKISTPFMKCPLLEDKTWDDDEEEMDDKLKKARDAERLAAKLRTIDDAERIAAKLRRVRVADIVERIEVCTVPFHNNNGCV BKK3 LKELLMTASAKISTPFMKCPLLEDKTWDD--EGMDDELKKVRDAERLAAKLRTVCDAERIAAKLRRVRVADIVERIEVCTVPFHNNNGCV

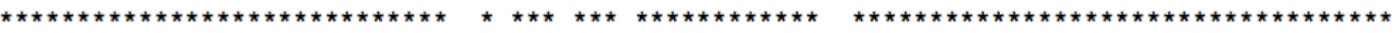

91 STLYKLQLKLYPQGLYPRQSELTVEECHETLRTVFIDAMDLAISKHLDLLHKINE IQAVKSNDMESQRSDGVEESENGPTDEDNGVSDGE 91 STLYKLQLKLYPQGLYPRQSELTVEECHETLRTVFIDAMDLAISKHLDLLHKINEIQAVKSNDMESQRSDGVEESENGPTDEDNGVSDGE 89 STLYKLQLKLYPQGLYPHQSELTVEECHETLRTVFIDAMDLAISKHLDLLHKINE IQAVRSNDMESQQSDGVEESENGPTDEDNGVSDGE

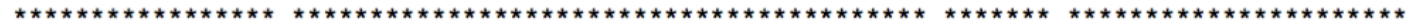

181 NEDDLGADAEKWKRQEIDEMEYDDDAEKEEGFDMDSESEEDTKSKPESEDHQAKLDEELEESEEGHVLDSSNKGENLKAKQATARLEDEM 270 181 NEDDLGADAEKWKRQE IDEMEYDDDAEKEEGFDMDSESEEDTKSKPESEDHOAKLDEELEESEEGHVLDSSNKGENSKAKQATARLEDEM 270 179 NEDDLGADAEKWKRQEIDEMEYDDDAEKEEGFDMDSESEEDTKSKPESEDHRAKLDEELEESEEGHVLDSSNKGENSKAKQATARLQDEV 268

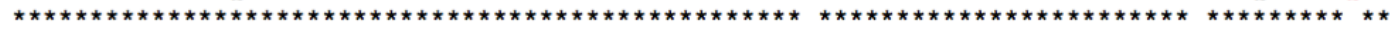

271 NEAEDEKAQVTIKFKKNIKWTIHYESTGLNFEVHYALQEQPHILLAQIAQRTARSVFVKACKNIDRCEVNKPKKIDNNTINTPITLQTAG 271 NEAEDEKAQVTIKFKKN IKWT IHYESTGLNFEVHYALQEQPHILLAQIAQRTARSVFVKACKNIDRCEVNKPKKIDNNTINTPITLQTAG 269 NEAEDEKAQVTIKFKKNIKWTIHYESKGLNFEVHYALQEQPHILLAQIAQRTARSVFVKACKNIDRCEVNKPKEIDNNAINTPITLQTAG

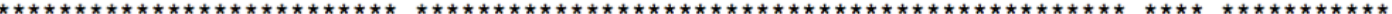

361 VNFEVFHKLVDYLDINEVRSNDIHAMLNTYGVEAARATIIGEVKGVFGAY 410 361 VNFEVFHKLVDYLDINEVRSNDIHAMLNTYGVEAARATIIEEVKGVFGAY 410 359 VNFEVFHNLVDYLDINEVRSNDIHAMLNTYGVEAARATIIEEVKGVFGAY 408

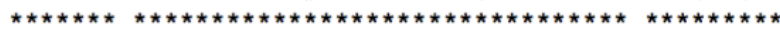

$\mathrm{BKKT}=0$. rufipogon

$\mathrm{BKK} 2=0$. longistaminata

$\mathrm{BKK} 3=0$. officinalis

B
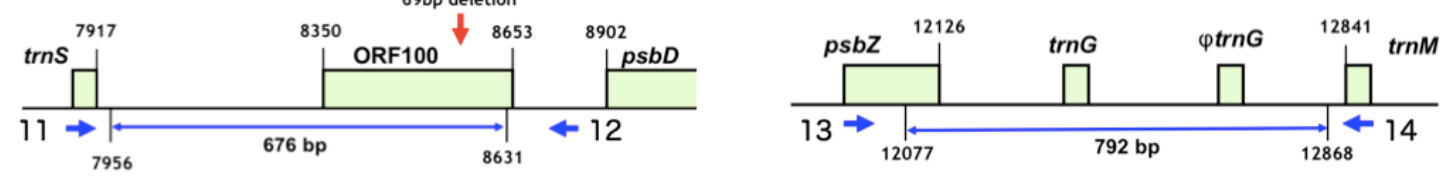

\begin{tabular}{|c|c|c|c|c|c|c|c|c|c|c|c|}
\hline \multirow[b]{2}{*}{ Species } & \multirow[b]{2}{*}{ Accessions } & \multicolumn{10}{|c|}{ ORF100 } \\
\hline & & 10 & 13_14 & 72 & 173 & 193 & 239 & 442 & 471 & 584 & 594-663 \\
\hline \multirow{2}{*}{$\begin{array}{l}\text { O. sativa japonica } \\
\text { O. sativa indica }\end{array}$} & Nipponbare & A & - & A & A & A & A & A & $T$ & C & 69 bp \\
\hline & A15 & - & - & - & G & - & - & • & • & - & - \\
\hline \multirow{2}{*}{$\begin{array}{l}\text { O. rufipogon (P) } \\
\text { O. rufipogon (A) }\end{array}$} & w1956 & • & - & $\cdot$ & $\cdot$ & $\cdot$ & $\cdot$ & $\cdot$ & • & $\cdot$ & 69 bp \\
\hline & w1958 & . & - & - & G & . & - & - & • & - & - \\
\hline \multirow{2}{*}{ O. longistaminata } & w0643 & $\cdot$ & - & $\cdot$ & $\cdot$ & $\cdot$ & $\cdot$ & $\cdot$ & $\cdot$ & $\cdot$ & 69 bp \\
\hline & w1413 & G & - & - & - & - & - & - & - & - & 69 bp \\
\hline BKK strain & & . & - & . & . & . & . & . & . & G & 69 bp \\
\hline O. officinalis & w0002 & $\cdot$ & A & $T$ & $\cdot$ & G & $T$ & G & G & ТСТTCT & 69 bp \\
\hline
\end{tabular}

\begin{tabular}{cccccccccc}
\hline \hline \multicolumn{10}{c}{ PSBZ } \\
\hline 12 & 52 & 136 & 409 & $427 \_428$ & 487 & 594 597 & 604 & 627 & 629 \\
\hline G & A & C & C & - & T & GGGA & G & G & A \\
$\cdot$ & $\cdot$ & $\cdot$ & $\cdot$ & - & $\cdot$ & - & $\cdot$ & $\cdot$ & $\cdot$ \\
\hline$\cdot$ & $\cdot$ & $\cdot$ & $\cdot$ & - & $\cdot$ & GGGA & $\cdot$ & $\cdot$ & $\cdot$ \\
$\cdot$ & $\cdot$ & $\cdot$ & $\cdot$ & - & $\cdot$ & - & $\cdot$ & $\cdot$ & G \\
\hline$\cdot$ & G & $\cdot$ & $\cdot$ & - & $\cdot$ & - & $\cdot$ & $\cdot$ & $\cdot$ \\
$\cdot$ & G & $\cdot$ & $\cdot$ & - & $\cdot$ & - & $\cdot$ & $\cdot$ & $\cdot$ \\
\hline$\cdot$ & $\cdot$ & $\cdot$ & $\cdot$ & - & $\cdot$ & - & $\cdot$ & $\cdot$ & $\cdot$ \\
\hline A & $\cdot$ & G & - & C & C & - & C & C & $\cdot$ \\
\hline
\end{tabular}

Figure 4. Alignment of three Ptag sequences and plastid DNA sequences in BKK strain. (A) Three Ptag sequences (BKK1, $\mathrm{BKK} 2$, and BKK3) of BKK strain were completely identical to that of O. rufipogon, O. longistaminata, and O. officinalis, respectively. (B) Two plastid sequences, ORF100 (676 bp) and psbZ(792 bp), were analyzed using primer 11 - 12 and 13 - 14, respectively. Only unique sites among $O$. sativa, $O$. rufipogon, $O$. longistaminata, $O$. officinalis and BKK strain were shown. Dot: same nucleotide, Bar: deletion. 
officinalis and BKK strain were digested by three different restriction enzymes and subjected to agarose gel electrophoresis. Southern blot pattern was obtained by hybridization with cloned CentO-C1 fragment as a probe (Figure 5(A)). Hybridization signals were clearly detected in $O$. officinalis and BKK strain but there is no signal in O. sativa.

\section{Discussion}

Akimoto et al. (1999) reported that presence of unusual big wild rice (named as BKK) strain having completely sterile spikelet and long-wide leaves in canals near the Wat Chalo temple in the Bang Kruai district, Bangkok (Figure 1) [23]. Although many plants similar to the BKK strain were grown around there, it considered that they were clonally propagated. Kuroda et al. (2003) reported that Chao Phraya basin was one of the most important regions to conserve wild Oryza species in mainland Southeast Asia because three Oryza species, O. rufipogon, $O$. officinalis and $O$. ridleyi habitat together [24].

The BKK strain was confirmed as triploid plant because its chromosome number was 36 while Oryza diploid species was $2 n=24$ (Figure 2(A)). Relative DNA content of leaf cells of $O$. sativa was compared to that of $O$. officinalis and BKK strain (Figure 2(B), Figure 2(C)). When relative DNA content of $O$. sativa was adjusted to $100, O$. officinalis and BKK strain showed 150 and 175 , respectively. Miyabayashi et al. (2007) reported that DNA contents of $O$. sativa and other AA genome species were similar and much lower than those of $O$. officinalis and other CC genome species [4]. This result suggested that two AA genome species and one CC genome species were responsible for the origin of BKK strain.

Nakamura (2016) found that protein tag (Ptag) sequence located in the C-terminal region of POLA1 subunit showed species-specific variation in not

A

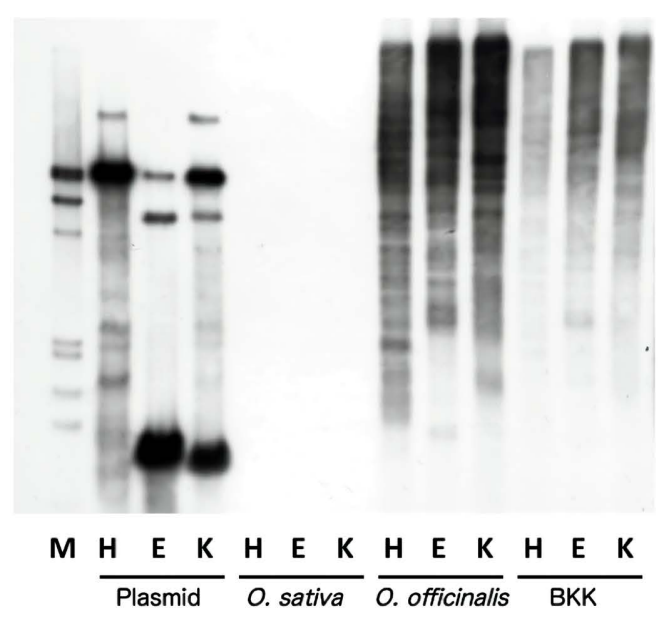

B

\section{O. longistaminata $\mathrm{x} \quad 0$. rufipogon}

$F_{1}$

Unreduced gametes of egg cell

x 0. officinalis

BKK strain

Figure 5. Southern blot hybridization of the CentO-C1 repeat in BKK strain. (A) Genomic DNAs of O. sativa, O. officinalis and BKK strain were digested by HindIII (H), EcoRI (E), and KpnI (K) and subjected to agarose gel electrophoresis. Blotted nylon membrane was hybridized with labelled CentO-C1 fragment (1.2 $\mathrm{kb})$. (B) Schematic representation for the origin of BKK strain. 
only plant but also fungi, animals and protists [13]. The Ptag sequences were highly conservative within a species but differentiated between species [10]. Because intron 19 and 20 sequences of PolA1 gene were located within Ptag-coding sequence (Figure $3(\mathrm{~A})$ ), these two intron sequences were also conservative in a species. Therefore, analysis of intron 19 and 20 sequences of PolA1 gene were useful to reveal ancestral species of polyploid species, such as Triticum-Aegilops [9], Triticum-Hordeum [10], and Brassica [11] [12].

Recent analysis of intron 20 of PolA1 gene indicated that S-type intron 20 $(0.14 \mathrm{~kb})$ was observed in $O$. sativa, $O$. rufipogon, $O$. glumaepatula and $O$. barthii whereas L-type intron $20(1.5 \mathrm{~kb})$ was found in $O$. longistaminata and $O$. meridionalis (Htut et al. submitted). Sequence analysis showed that S-type intron 20 was probably derived from L-type intron 20 due to large deletion by intramolecular homologous recombination between two TTTTGC repeats within L-type intron 20 of PolA1 gene (Htut et al. submitted). Because all Oryza species other than AA genome contained the L-type intron 20, this result suggested that $O$. rufipogon was originated from single or few individuals of $O$. longistaminata.

In this study, BKK strain contained three different intron 20 sequences, one S-type intron 20 (rufA $0.14 \mathrm{~kb}$ ) and two L-type intron 20 (lonA $1.5 \mathrm{~kb}$, offC 1.8 $\mathrm{kb}$ ) (Figure 3(B)). Sequence analysis showed that rufA sequence of BKK strain was identical to S-type intron 20 sequence of $O$. rufipogon. While lonA and offC sequences of BKK strain were grouped with L-type intron 20 sequences of $O$. longistaminata and $O$. officinalis, respectively (Figure $3(\mathrm{C})$ ). This result suggested that BKK strain contained genetic materials derived from O. rufipogon, $O$. longistaminata and $O$. officinalis. Three Ptag-coding sequences of BKK strain were also determined by direct sequence analysis using various sequencing primers (Table 2). Deduced three Ptag sequences (BKK1, BKK2 and BKK3) of BKK strain were identical to that of $O$. rufipogon, $O$. longistaminata and $O$. officinalis (Figure 4(A)). This result indicated that these three Oryza species were involved in the origin of BKK strain.

Two plastid, ORF100 and $p s b Z$, sequences of BKK strain were different from those of $O$. officinalis and similar to those of $O$. rufipogon and $O$. longistaminata (Figure 4(B)). Although plastid sequences of BKK strain did not consistent with those of $O$. rufipogon and $O$. longistaminata, cytoplasmic origin of BKK strain might be $O$. longistaminata because $O$. rufipogn perennial strain contained an unique GGGA insertion in the $p s b Z$ sequence.

Lee et al. (2005) reported that CentO-C1 repeats were specific to $O$. officinalis and CC genome species and absent in other Oryza species [21]. Southern hybridization showed that $\mathrm{CentO}-\mathrm{C} 1$ signals were present in $O$. officinalis and $\mathrm{BKK}$ strain but absent in O. sativa (Figure 5(A)). This result indicated that BKK strain contained genetic materials derived from $O$. officinalis.

Although BKK strain inhabited together with $O$. rufipogon and $O$. officinalis, $O$. longistaminata was known to be endemic to Africa. However, we found $O$. logistaminata-like cryptic species in Mekong Delta, Vietnam (Htut et al. submit- 
ted). And $O$. rufipogon was considered to be originated from $O$. longistamina (Htut et al. submitted). Therefore, it is possible that $O$. longistaminata was used to distribute in Southeast Asia.

Taken together of the results in this study, BKK strain probably originated as follow (Figure 5(B)). At first, an interspecific hybrid between $O$. longistaminata and $O$. rufipogon was occurred in the past. Then, hybridization between unreduced female gamete produced in the interspecific hybrid and male gamete from $O$. officinalis had produced natural triploid BKK strain. Therefore, it is interested that BKK strain maintained ancient genome of $O$. longistaminata, which was extinct in Southeast Asia.

\section{Conclusion}

We found wild rice (BKK) strain with big stature and sterile spikelet in Bangkok city of Thailand. The BKK strain is recognized as natural triploid by counting chromosome number and measuring relative DNA content of nuclei. Because Ptag sequence showed species-specific variation in eukaryotic species, Ptag sequence, encoded by exon $19-20$ of PolA1 gene, was analyzed. The result indicated that BKK strain contained three Ptag sequences, which was identical to that of $O$. longistaminata, $O$. rufipogon and $O$. officinalis, respectively. Sequence analysis for ORF100 and $p s b Z$ of plastid DNA showed that $O$. longistaminata was probably maternal parent. Contribution of $O$. officinalis was confirmed by Southern blot analysis using CentO-C1 probe specific to $O$. officinalis. These data indicated that BKK strain was originated by hybridizations among three parental species, $O$. longistaminata, $O$. rufipogon and $O$. officinalis.

\section{Acknowledgements}

We are grateful to Dr. Takato Koba, Chiba University, for his encourage and helpful advice on this research. The authors thank Sakura Science Exchange Program of Japanese governmental scholarship for the valuable supports of the first author.

\section{Conflicts of Interest}

The authors declare no conflict of interest regarding publication of this manuscript.

\section{References}

[1] Chu, Y.E. and Oka, H.I. (1970) Introgression across Isolating Barriers in Wild and Cultivated Oryza Species. Evolution, 24, 344-355. https://doi.org/10.1111/j.1558-5646.1970.tb01766.x

[2] Takahashi, H., Sato, T., Sato, Y-I. and Nakamura, I. (1990) Genome-Type-Specific Variation of the 19th Intron Sequence within the RNA Polymerase I Largest Subunit Gene in the Genus Oryza. Plant Systematics and Evolution, 282, 21-29. https://doi.org/10.1007/s00606-009-0172-x

[3] Xu, J-H., Osawa, I., Tsuchimoto, S., Otsubo, E. and Otsubo, H. (2005) Two New 
SINE Elements, p-SINE2 and p-SINE3, from Rice. Gene and Genetic Systems, 80, 161-171. https://doi.org/10.1266/ggs.80.161

[4] Miyabayashi, T., Nonomura, K.I., Morishima, H. and Kurata, N. (2007) Genome Size of Twenty Wild Species of Oryza Determined by Flow Cytometric and Chromosome Analyses. Breeding Science, 57, 73-78. https://doi.org/10.1270/jsbbs.57.73

[5] Seither, P., Croy, J.F., Pouska, A. and Grummt, I. (1997) Molecular Cloning and Characterization of the cDNA Encoding the Largest Subunit of Mouse RNA Polymerase I. Molecular Geneics and Genomics, 255, 180-186. https://doi.org/10.1007/s004380050487

[6] Kawahara, Y., Bastide, M. de la, Hamilton, J.P., Kanamori, H., Mccombie, W.R., Ouyang, S., Schwartz, D.C., Tanaka, T., Wu, J., Zhou, S., Childs, K.L., Davidson, R.M., Lin, H., Quesada-Ocampo, L., Vaillancourt, B., Sakai, H., Lee, S.S., Kim, J., Numa, H., Itoh, T., Buell, C.R. and Matsumoto, T. (2013) Improvement of the Oryza sativa Nipponbare Reference Genome Using Next Generation Sequence and Optical Map Data. Rice, 6, Article No. 4. https://doi.org/10.1186/1939-8433-6-4

[7] Zhang, X., Takahashi, H., Nakamura, I. and Mii, M. (2008) Molecular Discrimination among Taxa of Petunia axillaris Complex and P. integrifolia Complex Based on PolA1 Sequence Analysis. Breeding Science, 58, 71-75.

https://doi.org/10.1270/jsbbs.58.71

[8] Nakamura, I., Rai, R., Takahashi, H., Kato, K., Sato, Y-I. and Komatsuda, T. (2009) Aegilops Section Sitopsis Species Contains the Introgressive PolA1 Gene with a Closer Relationship to That of Hordeum than Triticum-Aegilops Species. Breeding Science, 59, 602-610. https://doi.org/10.1270/jsbbs.59.602

[9] Takahashi, H., Rai, R., Kato, K. and Nakamura, I. (2010) Divergent Evolution of Wild and Cultivated Subspecies of Triticum timopheevii as Revealed by the Study of PolA1 Gene. Genetic Resources and Crop Evolution, 57, 101-109.

https://doi.org/10.1007/s10722-009-9454-y

[10] Rai, B., Takahashi, H., Kato, K., Sato, Y.-I. and Nakamura, I. (2012) Single-Copy Nuclear PolA1 Gene Sheds Light on the Origin of S Genome with Relationships to B and G Genomes of Polyploid Wheat Species. Genetic Resources and Crop Evolution, 59, 1713-1726. https://doi.org/10.1007/s10722-012-9793-y

[11] Fareed, A., Shindo, H., Takahashi, H. and Nakamura, I. (2015) Analysis of PolA1 intron 19 and Ntag Sequences Reveals Two Ancestral Lineages in the Origin of the Brassica rapa Complex and Chinese Cabbage (B. rapa var. pekinensis). Journal of Horticutural Science and Biotechnology, 90, 273-278. https://doi.org/10.1080/14620316.2015.11513182

[12] Fareed, A., Shindo, H., Takahashi, H. and Nakamura, I. (2016) Phylogeny of PolA1 Gene Consistent with the Relationships of U's Triangle in Brassica. The Horticutur Journal, 85, 55-62. https://doi.org/10.2503/hortj.MI-052

[13] Nakamura, I. (2016) Method of Identifying Eukaryotic Species. JP 2016129518-A 358.

[14] Yamanishi, C., Alshahni, M.M., Sano, A., Nakamura, I. and Makimura, K. (2017) A New Marker Sequence for Systematics of Medically Important Fungi Based on Amino Acid Sequence of the Largest Subunit of RNA Polymerase I. Medical Mycology, 55, 555-562. https://doi.org/10.1093/mmy/myw098

[15] Fukui, K. (1996) Plant Chromosome at Mitosis. In: Fukui, K. and Nakayama, S., Eds., Plant Chromosome: Laboratory Methods, CRC Press, Boca Raton, 1-17.

[16] Mishiba, K., Ando, T., Mii, M., Watanabe, H., Kokubun, H., Hashimoto, G. and Marchesi, E. (2000) Nuclear DNA Content as an Index Character Discriminating 
Taxa in the Genus Petunia sensu Jussieu (Solanaceae). Annals of Botany, 85, 665-673. https://doi.org/10.1006/anbo.2000.1122

[17] Doyle, J.J. and Doyle, J.L. (1987) A Rapid DNA Isolation Procedure for Small Quantities of Fresh Leaf Tissue. Phytochem Bulletin, 19, 11-15.

[18] Takahashi, H., Sato, Y-I. and Nakamura, I. (2008) Evolutionary Analysis of Two Plastid DNA Sequences in Cultivated and Wild Species of Oryza. Breeding Science, 58, 225-233. https://doi.org/10.1270/jsbbs.58.225

[19] Thompson, J.D., Higgins, D.G. and Gibson, T.J. (1994) CLUSTAL W: Improving the Sensitivity of Progressive Multiple Sequence Alignment through Sequence Weighting, Position-Specific Gap Penalties and Weight Matrix Choice. Nucleic Acids Research, 22, 4673-4680. https://doi.org/10.1093/nar/22.22.4673

[20] Kumar, S., Stecher, G. and Tamura, K. (2016) MEGA7: Molecular Evolutionary Genetics Analysis Version 7.0 for Bigger Datasets. Molecular Biology and Evolution, 33, 1870-1874. https://doi.org/10.1093/molbev/msw054

[21] Lee, H.-R., Zhang, W., Langdon, T., Jin, W., Yan, H., Cheng, Z. and Jiang, J. (2005) Chromatin Immunoprecipitation Cloning Reveals Rapid Evolutionary Patterns of Centromeric DNA in Oryza Species. Proceedings of the National Academy of Sciences of the United States of America, 102, 11793-11798. https://doi.org/10.1073/pnas.0503863102

[22] Rogers, S.O. and Bendich, A.J. (1985) Extraction of DNA from Milligram Amounts of Fresh, Herbarium and Mummified Plant Tissues. Plant Molecular Biology, 5, 69-76. https://doi.org/10.1007/BF00020088

[23] Akimoto, M., Shimamoto, Y. and Morishima, H. (1999) The Extinction of Genetic Resources of Asian Wild Rice, Oryza rufipogon Griff.: A Case Study in Thailand. Genetic Resources and Crop Evolution, 46, 419-425. https://doi.org/10.1023/A:1008622405001

[24] Kuroda, Y., Urajrong, H. and Sato, Y-I. (2003) Population Genetic Structure of Wild Rice (Oryza rufipogon) in Mainland Southeast Asia as Revealed by Microsatellite Polymorphisms. Tropics, 12, 159-170. https://doi.org/10.3759/tropics.12.159 